\title{
Cathepsin K inhibitors: emerging treatment options for osteoporosis
}

\author{
Peter Pietschmann
}

Published online: 19 February 2015

(C) Springer-Verlag Wien 2015

Cathepsin $\mathrm{K}$ belongs to the group of cysteine proteases; most members of this family are widely expressed throughout many tissues and are involved in protein degradation and extracellular matrix remodelling. Cathepsin K has a relatively restricted expression pattern; it is highly expressed in the cells responsible for bone resorptionthe osteoclasts - and has a crucial role in the degradation of bone matrix, in particular type I collagen. In mice, the deficiency of cathepsin K leads to a high bone mass phenotype ("osteopetrosis") that resembles pycnodysostosis in humans. The relevance of cathepsin $\mathrm{K}$ for bone is also underscored by the observation that overexpression of the enzyme results in a low bone mass/"osteoporotic" phenotype. The fundamental role of cathepsin $\mathrm{K}$ in bone resorption has led to the development of cathepsin $\mathrm{K}$ inhibitors for the treatment of diseases characterized by excessive bone degradation. Currently, several cathepsin $\mathrm{K}$ inhibitors are under preclinical or clinical investigation for indications such as bone metastases, rheumatoid arthritis or osteoporosis [1].

This thematic issue of the Wiener Medizinische Wochenschrift deals with cathepsin $\mathrm{K}$ inhibitors as emerging treatment options for osteoporosis. In the first manuscript Reinhard Gruber gives a concise overview of the cell biology of the osteoclast and the molecular basis of bone resorption. In the next article Irene Ham- rick and co-workers review the current state of the art of the treatment of osteoporosis. The authors very convincingly demonstrate the effectiveness of the available treatments, but also point out that a large proportion of the patients with osteoporosis are not adequately treated. The latter fact clearly underscores the need for novel therapeutic options. Finally, Marion Gamsjäger and Heinrich Resch review preclinical and clinical data on cathepsin $\mathrm{K}$ inhibitors for the treatment of osteoporosis, in particular clinical studies on odanacatib and ONO-5334. According to a recent communication, a phase III study in women with postmenopausal osteoporosis ("LOFT") revealed, that odanacatib significantly reduced the risk of vertebral, non-vertebral and hip fractures (http://www. businesswire.com/news/home/20140915006286/en). It thus can be expected that in the near future the first cathepsin $\mathrm{K}$ inhibitor will be approved for the treatment of osteoporosis.

\section{Reference}

1. Pietschmann P, Föger-Samwald U, Sipos W, Rauner M. The role of cathepsins in osteoimmunology. Crit Rev Eukaryot Gene Expr 2013;23:11-26. 